\title{
LOS PROCESOS DE DISPUTA EN LA IMPLEMENTACIÓN DE POLÍTICAS EDUCATIVAS REFERIDAS AL TRABAJO EN EL NIVEL SECUNDARIO DE LA PROVINCIA DEL NEUQUÉN: EL CASO DE LOS PLANES DE MEJORA INSTITUCIONAL: UN ANÁLISIS PRELIMINAR
}

\author{
Natalia Noemí Fernández* \\ Universidad Nacional del Comahue, Argentina. \\ natinoe@hotmail.com
}

Recibido: 20/09/2016 Aceptado: 1/11/2016

\section{Resumen}

Estudiar la articulación educación y trabajo resulta fundamental para comprender las concepciones epistemológicas que subyacen en las políticas educativas, en especial, las que refieren a la 'educación para el trabajo'. Ante ello, es necesario analizar y complejizar las disputas que se juegan al interior de la concreción y materialización de dichas políticas. En este caso, nos referimos a los Planes de Mejora Institucional, en el marco de la ley de Educación Nacional, que es fruto de los lineamientos políticos y estratégicos de la educación secundaria obligatoria.

En este artículo se presentan los primeros avances de una tesis doctoral (1) en curso. Se analizan entrevistas semi estructuradas a informantes calificados con la intención de vislumbrar los sentidos que construyen algunos actores involucrados en la materialización de las políticas educativas. Se realiza un análisis cualitativo de los datos proporcionados por los sujetos intervinientes.

Magister en Política y Gestión de la Educación por la Universidad Nacional de Luján, doctoranda en Educación de la Universidad Nacional del Comahue. Docente e Investigadora de la Facultad de Ciencias de la Educación- UNCo. 
Palabras Clave: Política educativa - Planes de mejora - Educación para el trabajo - Escuela secundaria.

\section{Abstract}

To study education and work result essential to understand the conceptions of educational polices, specially, the ones that refer to "education for work". Therefore, it is necessary to understand the game inside of concretion and implementation of these polices. In that case, we refer to organizational improvement, within the framework of National Education law, which is the result of political and strategic guidelines of secondary school.

In this article, the first steps of a doctoral thesis are presented, analyzing semi structured interviews to qualified informants, with the intention of seeing the senses that some actors build in the educational polices implementation.

Keywords: Educational policy - Organizational improvement - Education for work - Secondary school.

\section{Introducción}

Estudiar la articulación educación y trabajo resulta fundamental para comprender las concepciones epistemológicas que subyacen en las políticas educativas, en especial, las que refieren a la 'educación para el trabajo'. Ante ello, es necesario comprender y complejizar las disputas que se juegan al interior de la concreción y materialización de dichas políticas. En especial, nos referimos a los Planes de Mejora Institucional (PMI), en el marco de la ley de educación nacional, que es fruto de los lineamientos políticos y estratégicos de la educación secundaria obligatoria.

Para realizar un diagnóstico de la situación de la Provincia de Neuquén, retomamos una investigación de Moschini (2013) que analiza, en su primera fase exploratoria, el estado de situación de los PMI en la provincia de Neuquén. La autora sostiene que los planes constituyen una redefinición del sentido de las políticas educativas impulsadas, persiguiendo la finalidad de dotar de recursos a los actores institucionales para la construcción de espacios alternativos que relocalicen, en el centro de las preocupaciones, a las trayectorias educativas de los estudiantes, sin dejar de reconocer los entrecruzamientos y disputas que estas pretensiones políticas, convertidas en experiencias y prácticas singulares, negocian con el núcleo estable del formato escolar tradicional. Esta investigación exploratoria nos ofrece indicios sobre el estado de situación provincial con relación a la política educativa nacional. Si bien el diagnóstico situacional es del 2013, es necesario aclarar que los planes de mejora siguen vigentes, conforme la Resolución proveniente del Consejo Federal de Educación N¹88/12 "Plan Nacional de educación obligatoria y formación docente", la cual se proyecta para el quinquenio 2012 -2016 inclusive. 
La relevancia de esta problemática radica en el abordaje de las políticas educativas referidas a la formación para el trabajo y los sentidos que expresan/construyen y legitiman, en la provincia de Neuquén, los actores involucrados en la materialización de la política en los quehaceres y prácticas escolares cotidianas. En este sentido, indagar acerca de las significaciones de los diferentes sujetos participantes, ofrece herramientas válidas para desnaturalizar las encrucijadas que existen entre la educación y el trabajo y las diversas posturas que hay en esta relación desde una visión crítica y de replanteo del lugar que tiene la formación para el trabajo en la escuela y sus lazos con el mundo del trabajo actual.

Recuperando los aportes de Ball (2002), concebimos la política como texto y como discurso; es decir, implica tanto las fuerzas que despliega el Estado sobre las instituciones, como las apropiaciones que hacen los agentes de ella.

En este artículo presentamos los primeros avances de una tesis doctoral en curso. En este caso analizamos tres entrevistas semi estructuradas suministradas a informantes calificados en el año 2014, articulados con la normativa nacional y provincial vigente, en la fase preliminar del proceso de investigación (3). Se realizó un análisis cualitativo de los datos.

Los informantes calificados fueron dos asistentes técnicos territoriales (ATT) que realizan el monitoreo de los planes de mejora en la provincia (PMI). Las asistentes técnico territoriales son docentes, en su mayoría, jubilados de la provincia, cuya relación contractual es por parte del ministerio de educación nacional para realizar un seguimiento pedagógico y administrativo de los planes de mejora en las escuelas secundarias comunes (4).

En el caso de las escuelas técnicas realizamos entrevistas a una supervisora jubilada y un supervisor en actividad. Estos insumos fueron necesarios e importantes para obtener un primer diagnóstico de lo que acontece en las escuelas de la provincia referido a las políticas educativas, en especial, aquellas ligadas a la 'educación para el trabajo' (5).

En el caso de los planes de mejora sólo focalizamos la mirada en aquellos que tengan algún vínculo con la formación para el trabajo, en función de los datos proporcionados por los informantes calificados. En este marco, la Resolución 088/09 del Consejo Federal de Educación establece los planes de mejora cuyos ejes persiguen la finalidad de acompañar a los estudiantes en el ingreso, trayecto y egreso del Nivel para cumplir con la obligatoriedad escolar que establece la Ley de Educación Nacional. Es importante destacar que en las escuelas secundarias comunes de la provincia, existe cierta ausencia de proyectos vinculados con el mundo del trabajo, ya que no es una línea prioritaria desde el ministerio de educación nacional. Con lo cual, existen pocos proyectos referidos a la línea de egreso y de permanencia que impliquen una puesta en escena que se vinculen con el mundo del trabajo. El interés, en su mayoría, de los proyectos escolares, radica en el ingreso y la titulación de los 
estudiantes vinculado a la retención estudiantil solamente (Hernández y otros, 2014).

Con relación al análisis que se deriva de las entrevistas a los informantes calificados, consideramos que el hilo articulador refiere a los vínculos que se establecen entre el ministerio de educación nacional con cada una de las escuelas respecto del financiamiento de los proyectos escolares. Para desmenuzar esta idea, organizamos el escrito en dos puntos centrales: El primer punto analiza los vínculos entre nación y las escuelas respecto de los planes de mejora y los puntos de tensión que se visualizan en su financiamiento. Se sostiene que existe una 'bajada de línea' de nación hacia las escuelas con relación a los lineamientos que fija nación, sin proceso de mediación por parte de la provincia, en la producción de políticas concretas respecto de lineamientos específicos jurisdiccionales. Para desglosar esta idea, se analiza el proceso de implementación de los PMI en las escuelas comunes y lo que acontece en las escuelas técnicas. Se evidencia una paulatina 'autonomía' por parte de las escuelas en la toma de decisiones que refieren a: la elaboración de proyectos escolares, como el rol del director en su función de gestor de los fondos para la ejecución de los planes, así también la modalidad de pago a los docentes a cargo de los proyectos. Consideramos que existe una progresiva institucionalización de nuevos circuitos de financiamiento que se derivan del vínculo entre nación y cada una de las escuelas.

En el segundo punto planteamos ciertas especificidades que se evidencian en las escuelas técnicas a partir de las entrevistas proporcionadas por los supervisores. En este punto planteamos las problemáticas que se suscitan respecto de la no reglamentación de las prácticas profesionalizantes; como también la valoración que se realiza del título técnico.

El conjunto de ideas que evocan los diferentes informantes calificados las podemos tensionar con la siguiente afirmación:

(...) Las políticas gubernamentales y las normas educativas inciden en el proceso pero no lo determinan en su conjunto (...) La norma educativa oficial no se incorpora a la escuela de acuerdo con su formulación explícita original. Es recibida y reinterpretada por un orden institucional existente y por diversas tradiciones pedagógicas en juego dentro de la escuela (Rockwell, 1995, p.13).

Es decir, los sentidos y reinterpretaciones de los sujetos que 'viven la escuela' se resignifican de diversos modos. Veamos a continuación con detalle.

\section{Los vínculos Nación- Escuela: los planes de mejora y los puntos de tensión en su financiamiento.}

Para comprender las tramas complejas que existen entre el ministerio de 
educación nacional con la provincia, es necesario aclarar que a nivel jurisdiccional existe la Resolución 1687/11 que emana el Consejo Provincial de Educación que establece el Plan Jurisdiccional, conforme a las acuerdos federales concertados en el marco de la obligatoriedad de la escuela secundaria, para el diseño y la implementación de los planes de mejora institucional, para el período 2007-2011. (6)

A continuación, destacamos en las voces de las ATT, los puntos de tensión que se observan con respecto a las 'demoras' de la provincia en la elaboración de un plan jurisdiccional, necesario para sostener los lineamientos que nación pretende en el nivel secundario, con relación a los planes de mejora institucional.

En palabras de las entrevistadas:

$\mathrm{J}$ : lo que pasa es que acá está como medio atrasado la reforma educativa, viene bastante rezagada (...)

E: y por qué?

$\mathrm{J}$ : porque falta el plan jurisdiccional.

M: porque cada provincia tiene que tener su propio plan

E: y acá no está?

M: no, no (...) cuando vino nación llamó la atención sobre ese punto que yo entiendo que ya estaría hecho de antes pero no se ha hecho, por qué no lo han hecho? no sé

$\mathrm{J}$ : es raro porque cuando se firma el plan este quinquenal ya sabían que lo tenían que hacer porque fue refrendado... (Entrevista a las ATT).

Esta cita pone en evidencia la 'demora' en la elaboración del plan jurisdiccional y se subraya la idea de 'atrasos' a nivel provincial. Lo que resulta interesante, en el relato de las entrevistadas, es la 'llamada de atención' desde nación hacia la provincia, lo cual implica el otorgamiento de ciertas atribuciones por parte de la primera a la segunda, respecto de seguir con los acuerdos federales, con los lineamientos planteados por nación. Esto da cuenta cómo la provincia 'adhiere' a los acuerdos federales, sin embargo, no existe un nuevo plan jurisdiccional para el período posterior al 2011. Aspecto que imprime las particularidades de la provincia en sus 'atrasos', como refieren las entrevistadas.

Podemos conjeturar que los planes de mejora constituyen una estrategia clave de la política educativa nacional, habilitando a los actores escolares a promover acciones tendientes a 'garantizar' la permanencia de los estudiantes en el nivel, entro otros de sus postulados.

De este modo, inferimos que la provincia 'resuelve' las demandas que el ministerio de educación de la nación fija, mediante los acuerdos federales, en el cual, la provincia adhiere y reglamenta en función de determinados criterios federales, pero no desde objetivos y prioridades educativas a nivel provincial. En otras palabras, la política educativa provincial ha adoptado la forma de 
'dejar hacer': no censura pero tampoco produce políticas concretas en algún sentido al no existir alguna normativa provincial que fije y/o coordine lineamientos propios que refieran a las particularidades que asume la provincia (Hernández y otros, 2014).

En segundo lugar, sostenemos que el vínculo nación- provincia se materializa en las escuelas, a partir del financiamiento que provee nación a cada una de ellas según los proyectos presentados, donde nación es quien los evalúa y aprueba. La dificultad que se percibe son los tiempos concretos en la rendición de los fondos.

En palabras de un supervisor:

Hay otro problema es que los fondos no llegan a las escuelas pero eso es una cuestión netamente política. Los planes los aprueba nación, ellos tienen todo el equipo técnico para aprobar los planes. Lo que cuesta es que lleguen los fondos a las escuelas porque es decir hay unas diferencias en tiempos de la presentación del proyecto, la evaluación y el giro de fondos a la provincia por transferencia y que la provincia no te deja a vos como director más de 30000 pesos por transferencia y si tenes un torno por más de 150000 pesos cómo haces? (...) pero claro estamos en la pelea porque yo también me adhiero en que no lo podés comprar en cuotas, hay cosas que son necesarias entonces eso hay que resolverlo, cómo? Con los fondos de la provincia.

Como primer punto se destaca el manejo de los fondos que lo vincula a una cuestión política, ¿a qué se refiere? ¿A los tiempos en la asignación de recursos? Aquí queda al descubierto las particularidades que asumen el circuito y el giro de fondos. El vínculo que parece prevalecer, respecto del financiamiento, es 'nación- escuela' donde solamente cobra relevancia la provincia cuando existen 'emergentes' a resolver. En este sentido, la figura del director cobra relevancia en la toma de decisión sobre el destino de los recursos, quien 'resuelve' los emergentes, teniendo autonomía y poder de decisión. Existe una preeminencia en la función del director como 'gestionador' de los fondos.

Grinberg (2014) ofrece un interesante análisis para pensar el rol de la escuela, donde cae la responsabilidad en el sujeto en las formas de regular y gestionar las instituciones. La autora afirma:

Del mismo modo que cuando se remite a las escuelas en tanto organizaciones llamadas a gestionar y revertir las situaciones que viven a diario, en este caso son los individuos los que deben producir una reforma de sí a los efectos de enfrentar contiendas sociales

(...) En tiempos donde el empoderamiento orienta la vida de las instituciones, donde son las voluntades individuales aquellas que ponen en marcha y movilizan la vida diaria de, en este caso, las escuelas, una situación que inhibe esa voluntad deja el proyecto en su desnudez. (Grinberg, 2014, pp. 212-213). 
La gestión de la vida escolar y las propuestas pedagógicas recaen en el individuo que, en nuestro caso, es la figura de la dirección quien provee y distribuye los recursos para sobrellevar el cotidiano escolar.

Por otro lado, otra cuestión que se destaca en la cita del entrevistado es la demora en el otorgamiento de los fondos. Demora que también se visualiza en la entrevista a las ATT, en este caso, se traduce la misma como 'reclamo' por parte de los docentes:

...y por ahí los reclamos van en la parte administrativa hay falencias, que no les llega el dinero porque ellos tienen que pagar a los docentes entonces que ocurre que cuando no le depositan no tienen plata para pagar a los docentes y si la escuela tiene dinero a lo mejor lo sacan para pagar y después reponen pero viste es mucha plata entonces eso, de las horas institucionales, gastos operativos entonces es un problema. (Entrevista a las ATT).

Esta cita da cuenta de ciertos reclamos ante la demora en el otorgamiento de los recursos (7), donde es la escuela, o bien decir es el cuerpo directivo, quien tiene que pagar a sus docentes, como una función aditiva en su rol de 'gestor'.

Con respecto al financiamiento de las gestiones institucionales podemos decir que existe un vínculo entre el ministerio de educación de la nación con cada una de las escuelas. Sostenemos que existe una progresiva institucionalización de nuevos circuitos de financiamiento del sistema educativo, donde el financiamiento se materializa en cada una de las escuelas. Esto habilita futuras consideraciones acerca de los posibles modos de gestionar o distribuir los fondos, tanto al interior de las instituciones como a nivel macro, en el sentido de pensar los circuitos o mecanismos a nivel de la función o rol del Consejo Provincial de Educación (8) en la distribución de fondos.

Pudimos observar, en los relatos de las entrevistadas, que existiría cierto margen de decisión institucional para realizar una lectura de la propia situación escolar.

A su vez, existe otro nivel de dificultad en el proceso de implementación de los PMI, en cuanto al financiamiento de los proyectos escolares y la modalidad de pago a los docentes.

En palabras de las entrevistadas:

$\mathrm{J}$ : y los planes de mejora fue con mucha dificultad (...) y ahí fue como muy solapado porque ahí dijeron que había un dinero que iba a venir de nación y que la gente este por ejemplo había la profe de lengua y literatura que estaba a cargo del departamento trabajaba demasiado, le pagaban por seis horas pero trabajaba un montón tenía muchos alumnos entonces le dije que como nunca le habían dado la doble jefatura le dijeron que por qué no hacía un proyecto mas o menos para recibir plata y trabajar esas horas demás les iban a pagar así. (Entrevista a las ATT). 
Un aspecto a destacar es el modo de financiar proyectos que se realizaban en la institución pero que no tenía un 'reconocimiento' monetario hacia el docente. Podríamos decir que los planes vendrían a reconocer aquello que se hacía 'ad honorem' por parte de algunos docentes, entonces puede pensarse como 'motor de atracción' la aceptación de los planes en la escuela. Asimismo, cabría pensar ¿qué implicancias tiene este financiamiento en el trabajo docente? ¿cómo pensar este reconocimiento? ¿qué opinan los docentes sobre esto?. Aspectos para seguir profundizando.

Las políticas nacionales, como los PMI, en muchos casos permiten darle un carácter legal, dotar de recursos y formalizar proyectos que ya estaban en marcha en las escuelas.

Por otro lado, también se destacan ciertas resistencias de los docentes en la aceptación de los planes en las escuelas:

M: yo tengo una en este momento (nombra la escuela) que en reunión de la escuela dijeron que no, yo los cité, la supervisora también y bueno ahora van a convocar a otra reunión.

$\mathrm{J}$ : lo que pasa es que muchas veces recae en dos o tres personas la decisión y no convocan por ahí.

M: en la gestión de las conducciones anteriores la persona que conducía parece ser que no lo abrió como dice ella, se enteraron pocos, no hubo una apertura y tuvo una resisntencia. Ya de por sí vino una resistencia de que es plata en negro que no tiene aportes ese es un tema y que el director no lo abrió al consenso tuvo mas resistencia.

$\mathrm{J}$ : depende de la conducción también.

M: más obviamente el sindicato se opone a todo lo que el docente cobra sin tener aportes por todo eso". (Entrevista a las ATT, el resaltado es nuestro).

En esta cita se plantea el rol de la conducción en la apertura, o no, en la convocatoria por participar en los PMl a los docentes. A su vez, se evidencia los procesos de resistencia de algunos docentes, en una de las escuelas, con relación al modo de financiamiento de los planes, que tiene que ver con un reconocimiento monetario pero es 'en negro', es decir, es un reconocimiento sub-valorado. Ante esto, aparece otra figura clave que es el sindicato docente y la oposición a estas prácticas, quienes denuncian que genera cargos docentes por fuera del estatuto actual y habilita la incompatibilidad de cargos docentes, formalizando un trabajo precario (Barco y otros, 2012).

Del mismo modo, se evidencia que los procesos de implementación de los planes son diferentes en cada escuela, lo que genera cierto grado de autonomía en la toma de decisión de aceptación, o no, de los planes.

Si profundizamos lo que acontece en las escuelas técnicas, las dificultades en la demora en la concreción de los fondos son similares.

En palabras de una supervisora: 
En las escuelas técnicas hay planes de mejora, yo he participado siendo docente, el problema es que el dinero llega un año después. También tenes proyectos que financia el INET pero que son más difícil de ganar y el financiamiento es mucho más rápido. En el INET para el financiamiento pueden participar tanto las escuelas públicas como las privadas, hay que presentar un proyecto íntegro, presupuestar todo, cada uno de los detalles y con tres presupuestos diferentes (...) después lo que pasa es que esto lo tenes que presentar ponele en marzo y te contestan si ganaste en diciembre, con lo cual no podes comprar lo que proyectaste porque tarda como 9 meses pero si ganaste, la plata como mucho tarda dos tres meses en llegar, en cambio los planes de mejora demoran muchísimo más. (supervisora de escuela técnica).

Aquí se destaca la demora en la asignación de los fondos. A su vez, resalta una diferencia entre los planes de mejora y los proyectos que provienen desde el Instituto Nacional de Educación Tecnológica (INET). Distinción que cobra vigencia el financiamiento y los tiempos en hacerse 'efectivo' el giro de fondos, siendo los proyectos desde el INET más acelerados en la entrega de los recursos. Un aspecto a tener en cuenta, en esta distinción que realiza la supervisora, es cierto desconocimiento en la regulación de los planes de mejora, para las escuelas técnicas, por parte del INET. Respecto de la normativa, podemos mencionar, por ejemplo, la resolución 175/12 del Consejo Federal de Educación, el cual especifica los lineamientos y estrategias de los planes de mejora, en el marco de la ley de educación técnico profesional.

Otro aspecto para analizar son los proyectos que tienen que elaborar las escuelas para abastecer equipamiento y recursos materiales. La promoción en la elaboración de dichos proyectos proviene del ministerio de educación nacional a través del INET.

En palabras de la supervisora:

Por ejemplo, en química la idea es establecer laboratorio para análisis de agua, bacteriologización y esto los habilita a las escuelas a prestar servicios a terceros, hacia la comunidad el tema es que los docentes hacen todo este trabajo ad honorem, no les pagan un mango pero hay docentes que si lo hacen porque hay que sostenerlo. Es decir, la plata del INET es para la escuela, no para los docentes, y los chicos por supuesto. (supervisora escuela técnica)

Este relato es rico en cuanto se destacan varios aspectos, en primer lugar el recurso económico, que proviene desde el INET, para abastecer proyectos escolares que se vinculen con el equipamiento. En segundo lugar, se visualiza la doble carga en manos de los docentes para llevar a cabo los proyectos sin percibir alguna remuneración. Es interesante recuperar una frase de la entrevistada cuando plantea la necesidad de 'sostener' los proyectos, queda en manos de la voluntad y predisposición de los docentes en implementar y llevar a cabo tales proyectos. 
En tercer lugar, estos proyectos brindarían la posibilidad de prestar servicios a terceros como modo o acercamiento a la comunidad, habría que profundizar qué implicancias brinda estos 'servicios' y si es un nuevo u otro modo de financiar aquello que la escuela otorga.

Por otro lado, el supervisor plantea la demora en hacerse efectivo el financiamiento de los proyectos en las escuelas, dejando al descubierto los circuitos/mecanismos que se ponen en juego en la rendición de los recursos.

El supervisor plantea lo siguiente:

Nosotros dependemos de la dirección provincial de escuela técnica y la reglamentación básica del INET son los programas nacionales y los fondos que da. Te da la autonomía de cada escuela en hacer proyectos de diferentes líneas: equipamiento, horas institucionales a través de las tutorías como el proyecto tutorial que tienen las escuelas comunes con los PMI.

Se pone en evidencia cierto margen de decisión institucional para realizar una lectura de la propia situación escolar y de diagramar propuestas escolares sustentadas en la elección de estrategias específicas para el acompañamiento de sus estudiantes. A su vez, el entrevistado plantea el financiamiento de los proyectos desde el INET donde, una de las líneas, son los proyectos referidos al equipamiento de recursos materiales para las escuelas (9). Podemos observar que el circuito que prevalece, respecto del giro de fondos, es desde nación (a partir del INET) con cada una de las escuelas. En este circuito, la figura que cobra preeminencia es la del director como gestionador de los fondos asignados. Entonces cabría pensar nuevos circuitos de financiamiento a las escuelas y en esto nos preguntamos ¿cuál es el papel de la provincia, como instancia mediadora, entre nación y escuela? ¿Cuáles son las acciones, -si las hay-, que la provincia lleva a cabo a nivel jurisdiccional? Aspectos para profundizar en la investigación.

El rol de gestor en las direcciones de las escuelas otorga nuevas atribuciones a los sujetos que le permitirían controlar sus presupuestos y los procesos internos de toma de decisiones (Ball, 2007) (10). Arista necesaria para analizar los mecanismos que se ponen en juego tanto a nivel de la política educativa y los efectos que produce en los sujetos, en este caso, la escuela.

\section{Las escuelas técnicas: el reconocimiento del título y el quehacer de las prácticas educativas.}

En las entrevistas suministradas a los supervisores de escuelas técnicas se destacan dos puntos: la no reglamentación de las prácticas profesionalizantes (11), lo que conlleva diversas formas de 'solucionar' las mismas en las escuelas. La Ley de Educación Técnico Profesional plantea la necesidad de desarrollar oportunidades de prácticas profesionalizantes, donde las instituciones puedan generar estos espacios pero no se plantea de modo necesario 
u obligatorio (12).

Sobre esto, en una de las entrevistas a la supervisora se presentan las diferencias de lo que acontece en una escuela pública de gestión estatal y en una de gestión privada.

Por otro lado, también se acentúa cierto imaginario social respecto del título técnico en la impronta por su salida laboral, como 'garantía' al acceso al mercado de trabajo, lo que conlleva demandas desde las propias familias como desde el mundo del trabajo.

Respecto del primer punto, el supervisor nos comenta:

...el tema de la práctica profesionalizante todavía en la provincia no está reglamentada por ejemplo yo no pude disponer de 15 alumnos de petróleo, sabiendo la demanda que tenes de petróleo, no los puedo sacar porque por ejemplo la abogada que daba en el colegio la materia de organización laboral era la presidenta del colegio de abogados de cutral có y no aceptaba que se fueran los chicos a trabajar porque consideraba que con las practicas profesionalizantes se generaba un vínculo laboral, era su opinión porque en la línea de pensamiento era abogada y asesora de una empresa y como no está reglamentada en la provincia entonces había chicos que no lo hacían entonces algunos se agarraban de esa línea.

El relato expresa la ausencia en la reglamentación de las prácticas profesionalizantes, lo que conlleva ciertas tensiones con relación a las concepciones y las diferentes posturas de los docentes respecto de este tema. Por un lado se destaca la idea de realizar prácticas por 'fuera' de la escuela al expresar 'no los puedo sacar..., lo que imprime los límites y reglamentaciones al interior de la escuela con el 'exterior', con el afuera. Por otro lado se pone en evidencia el vínculo laboral que se genera en una situación de práctica laboral. ¿qué consecuencias conlleva esto en la relación empleador- estudiante? ¿cuáles son los derechos y obligaciones de los estudiantes con la empresa? Aspecto muy discutido en el ámbito escolar.

A su vez, al no existir una reglamentación explícita, se pone en evidencia las prácticas que se generan en las propias escuelas, algunas las llevan adelante, otras no, lo que expresa diferencias en la formación que reciben los estudiantes.

En palabras de una supervisora:

...te puedo hablar de la $X$ (nombra escuela de gestión estatal) y del Y (escuela de gestión privada) que tienen pasantías. En la $X$ sólo es para 6 alumnos, con los mejores promedios y dura 2 meses la pasantía, les pagan el pasaje de colectivo pero no es remunerada, el vice y el director son los que buscan los contactos con las empresas y ellos son los que te dicen el perfil de alumno que buscan y ahí se ve a quien se lleva, siempre son los que tienen mejores promedios, no es obligación hacer las pasantías pero si 
vos aceptaste tenes que terminarla con todo lo que implica: horarios a cumplir, tareas, roles a todos se les pide un trabajo final donde demuestre para qué les sirvió la experiencia. Son muy lindos los trabajos que han hecho y las empresas a veces también les solicitan trabajos. En la X se encargan el vice y el director, no había un docente a cargo de las pasantías. En cambio en el $Y$ las pasantías eran para todos y duraba 3 meses y habían docentes que se encargaban de gestionar las empresas. Fijate la diferencia, en una escuela pública y en una privada. Yo creo que hay una decadencia total en los últimos años, los docentes y directivos cada vez están más desgastados por todo, por ejemplo el arreglo de las escuelas y mantenimiento empieza en febrero cuando empiezan las clases, también está la presión del gremio porque siempre está lo salarial por ejemplo yo soy afiliada pero vez que está el caso Fuentealba I, Fuentealba II y lo salarial, se lucha por la defensa de la escuela pública y lo salarial. Siempre está.

La entrevistada nos comenta detalladamente la modalidad de las prácticas educativas en ambas instituciones, donde una es pública de gestión estatal y la otra es de gestión privada, dando cuenta de las oportunidades diferenciales que se destacan en la formación de los estudiantes, a saber: los criterios de selección de los estudiantes: en la pública acceden quienes tienen mejores promedios y en la privada lo realizan todos los alumnos; quienes se encuentran a cargo de gestionar las prácticas: en la primera es el equipo directivo, en la segunda hay un docente a cargo; y el tiempo de duración es otro indicador: en la primera dura dos meses, en la segunda tres meses.

Un aspecto que merece destacarse es el vínculo que establece la entrevistada entre las prácticas educativas diferenciales en la escuela pública y en la privada, con la 'decadencia' del sistema público, aludiendo al 'desgaste' de los docentes y a diversos indicadores tales como: condiciones edilicias y la 'presión del gremio' ¿a qué hace referencia? La entrevistada pareciera vincular la lucha salarial docente con la organización diferencial de las prácticas educativas en las escuelas. Este aspecto da cuenta que, al no existir una reglamentación de las prácticas, estas se ven supeditadas a las gestiones internas de cada una de las escuelas, dejando entrever las oportunidades diferenciales que se establecen en una escuela pública y en una privada.

Con respecto a la impronta del título técnico, se plantean demandas concretas por parte de las familias para que sus hijos asistan a esta modalidad.

La supervisora nos comenta:

$\mathrm{E}$ : y por qué crees que se elige la escuela técnica?

A: es por los padres, en muy pocos casos son los chicos, porque los padres están convencidos de que salen y tienen trabajo pero no saben si el chico tiene las habilidades técnicas necesarias porque si no las tiene es difícil que transiten por la técnica y después convencerlo al padre para que 
vaya a un CPEM, porque hay mucho innato pero también aprendido pero si no tienen lo innato no va. Porque hay muchos chicos que les gusta las ciencias sociales, o escuchar música, pero si te das cuenta que no va, listo, cambialo.

La elección de la escuela es por los padres que se reduce a la importancia en la salida laboral. El título técnico vendría a 'solventar' esta cuestión. A su vez, la entrevistada recupera dos aspectos: por un lado los intereses de los padres y, por otro, la necesidad que el chico tenga ciertas 'habilidades técnicas' como algo 'innato' que debe poseer el estudiante, como requisito 'necesario' para acceder a la modalidad, cuestión que si no lo posee, tendría que transitar un CPEM (13), realizando una valoración diferencial en la formación de las escuelas comunes con la técnica, jerarquizando esta última.

A su vez, la modalidad técnica parecería tener cierta especificidad, cierta adquisición de habilidades técnicas en la formación, de saberes específicos para la obtención de una especialización.

En palabras de los supervisores:

Existe una especificidad en cada uno de los títulos, hay mayor salida laboral que en un CPEM porque éste es muy light, es más débil en cuanto a su salida en cambio en las técnicas pueden trabajar en algo más específico como en petroleras, laboratorios, bromatología, en industrias de alimentos, tienen más variedad. (Entrevista supervisora).

"Las escuelas técnicas tienen más salida laboral que las otras por la especialización que son netamente de producción entonces vos tenés un técnico que sale con el título de técnico que esa es la gran demanda que tenemos ahora".(Entrevista a supervisor).

Los entrevistados destacan la impronta del título técnico en la salida laboral a partir de brindar una especialización para el mundo del trabajo. Especialización demandada ¿por la comunidad educativa? ¿por las empresas? Se puede pensar que las demandas vienen desde ambas partes, como aspecto a profundizar.

En este punto se observa la especificidad que pareciera aportar el título de técnico, lo que llevaría a obtener mayores oportunidades en el mundo del trabajo, donde el mercado laboral parecería estar demandado por requerir ciertas especificidades para las ramas de actividad. Sobre esto, se observa cierto reconocimiento del título en la adquisición de habilidades técnicas, de saberes específicos en la formación. Reconocimiento demandado por las familias en el acceso a esta formación por ser 'garantía' para una salida laboral, lo que construye un imaginario social en la valoración que se realiza al título técnico. 


\section{Conclusiones provisorias}

A partir del análisis de las tres entrevistas suministradas a informantes calificados (ATT y supervisores) pudimos evidenciar que en los vínculos entre nación y provincia, respecto de la política educativa, se destacan varios aspectos: la 'demora' de la provincia en la elaboración del plan jurisdiccional para continuar con los lineamientos político- pedagógicos propuestos por nación. Aún así, los planes de mejora se siguen sosteniendo en las escuelas. Sobre esto, el vínculo que se establece es entre nación con cada una de las escuelas a partir del control y regulación de los planes y el otorgamiento de financiamiento. Aquí cobra relevancia una figura clave que es el director quien gestiona los recursos asignados. Podemos conjeturar que existe cierta autonomía escolar en la elaboración de los planes y en la toma de decisiones con respecto al destino de los fondos. Sostenemos que existe una progresiva institucionalización de nuevos circuitos de financiamiento del sistema educativo, donde el financiamiento se materializa en cada una de las escuelas. Ante esto, ¿Cuál es el rol que asume el CPE, como órgano administrativo y regulador de la educación en esto? Sostenemos que existe una continuidad con las políticas de reforma educativa neoliberal en tanto la escuela es vista como unidad responsable de generar proyectos para resolver problemas que exceden a las escuelas y a sus colectivos docentes.(Barco y otros, 2012).

A su vez, queda al descubierto las demoras en el otorgamiento del financiamiento, donde es el director quien tiene que 'solucionar' los emergentes cotidianos y decidir el destino de los fondos.

Otro elemento es la función asignada al director, en el marco de la ejecución de los planes de mejora, y su relación con las autoridades provinciales (ya sea a través de los supervisores, o bien si existen otras figuras que emergen a partir de la implementación de esta política, como aspecto para profundizar).

En el caso de las escuelas técnicas, sucede lo mismo, donde la gestión de los fondos queda en manos del director. A su vez, no existe una reglamentación explícita de las prácticas profesionalizantes. La decisión queda supeditada a cada escuela, donde hay algunas que la realizan, otras no, argumentando las dificultades en sostener un vínculo laboral entre las empresas con los estudiantes. Consideramos que existen oportunidades diferenciales en la formación de los estudiantes respecto de este tema, donde se observan diferencias sustanciales de las prácticas educativas en una escuela pública y en una privada.

En este trabajo quisimos poner en evidencia cómo operan las normativas en los sentidos y prácticas que le otorgan los sujetos: los procesos de implementación, sus alcances y límites, en otras palabras, cómo se reinterpreta la norma en contextos particulares.

(...) Que una norma sea sancionada habla de que ciertos sentidos lograron instalarse en el espacio público, pero no implica necesariamente que esta 
construcción de sentido sea compartida por la mayoría de la opinión pública. La norma, cumple un rol central en la construcción de estos sentidos señalando nuevos límites y posibilidades. (Arroyo, 2012, p. 4)

En nuestro caso, podemos pensar que las regulaciones formales no poseen un gran impacto en el ordenamiento de las escuelas en la provincia que, por el contrario, se sostienen sobre sus particularidades, quehaceres cotidianos que van delineando su cultura institucional.

Si bien presentamos un panorama político educativo provincial que se ancla en el año 2014, las normativas analizadas siguen vigentes, aún queda pendiente analizar la coyuntura política educativa nacional y el futuro de los planes de mejora para el próximo año. Sin embargo, en este escenario, resulta fértil desarrollar algunas preguntas para seguir el análisis: ¿Cómo decodifican los actores institucionales las regulaciones estatales en la cotidianeidad escolar? ¿Qué lugar le asignan los actores a las reglamentaciones para pensar sus prácticas cotidianas? ¿qué efectos concretos producen las reglamentaciones?

Sostenemos que el gobierno provincial deja librado a cada institución la implementación o no de los PMI y enuncia declarativamente, como política provincial, la construcción de un proyecto jurisdiccional sin ser sostén de lo que acontece en las escuelas.

A su vez, observamos ciertas ausencias de normativas que regulen de modo específico lo que sucede a nivel jurisdiccional.

Reflexionar sobre el sentido de la escuela pública y las políticas educativas para los adolescentes, qué trayectorias se piensan, como pensar el vínculo con el trabajo es una discusión necesaria para pensar para qué proyecto de provincia se inscriben y bajo qué intereses.

\section{Notas bibliográficas}

(1) Título de la tesis provisorio: Los procesos de disputa en la implementación de políticas educativas referidas a la educación y al trabajo en el nivel secundario: el papel de los actores involucrados (Estado, sector empresarial y comunidad educativa) en la Provincia de Neuquén". Facultad de Ciencias de la Educación- Universidad Nacional del Comahue-.

(2) Resolución del Consejo Federal de Educación № 88/09 que aprueba el documento: "Institucionalidad y fortalecimiento de la educación secundaria obligatoria -Planes Jurisdiccionales y Planes de mejora institucional". Los mismos fueron implementados en la provincia de Neuquén de modo progresivo a partir del año 2010 en instituciones seleccionadas hasta abarcar la cobertura planificada. Según Resolución del Consejo Provincial de Educación (CPE) N0362/10 adopta las resoluciones del Consejo Federal de Educación N86/09 y 88/09 que acuerda criterios compartidos referidos a la propuesta de educación secundaria en su conjunto y a las metas comunes para la organización del nivel en la jurisdicción provincial. 
(3) Esta producción constituye un primer acercamiento al objeto de estudio para analizar posibles disputas/tensiones entre la norma nacional y la norma provincial. Las entrevistas a los informantes calificados se realizaron en el año 2014.

(4) Al momento de realizar la entrevista (2014), cada ATT tenía a su cargo alrededor de 9 escuelas secundarias comunes.

(5) Nos referimos a las formas de vinculación que propone, realiza o genera la escuela con el mundo del trabajo. En esta relación, encontramos tanto las herramientas intelectuales y de contenidos que transmite/enseña la escuela como las experiencias que habilita para que el sujeto comprenda e intervenga en su realidad actual. (Fernández, N, 2013)

(6) Al momento de realizar las entrevistas y su análisis, esta resolución seguía en vigencia

(7) Se otorga el financiamiento por proyectos escolares adheridos a los PMI que se vinculen con el acceso, permanencia y/o egreso de los estudiantes.

(8) Como ente autárquico, responsable de organizar, dirigir y administrar la educación de las escuelas en la provincia.

(9) Aún queda por analizar los lineamientos políticos y pedagógicos referidos a la capacitación de los docentes.

(10) El autor analiza las políticas de privatización de la escuela y en la escuela pública, donde el rol de gestor en las escuelas es clave para pensar estos modos de financiamiento. En este caso recuperamos la figura que adquiere la conducción o los equipos directivos.

(11) No existe normativa vigente a nivel provincial sobre la reglamentación de las prácticas profesionalizantes. Sólo encontramos la Resolución 1199 emanada desde el Consejo Provincial de Educación que data del año 1999 sobre las pasantías "como extensión orgánica del sistema educativo".

(12) Nos referimos al artículo 6 inciso c) de la LETP (Ley 26.058)

(13) Centro Provincial de Enseñanza Media (escuelas comunes)

\section{Referencias Bibliográficas}

- Arroyo, A. (2012). La performatividad de los procesos de regulación normativa. La norma como espacio de disputa por la direccionalidad de las políticas educativas para el nivel secundario. En VII Jornadas de sociología de la Universidad Nacional de La Plata. 5 a 7 de diciembre.

- Ball, S. (2007). Privatización encubierta de la educación. Internacional de la Educación. V Congreso Mundial. Bruselas: Instituto de educación. Universidad de Londres.

- -------- (2002) Grandes políticas, un mundo pequeño: Introducción a una perspectiva internacional en las políticas educativas. En Narodowski, M; Nores, M y Andrada, M (eds.) Nuevas tendencias en política educativa. Buenos Aires: Granica.

- Barco, S.; Dubinowski, S.; Cipresi, R.; Laurente M.; Rojo, R.; Junge, G. (2012). Políticas Educativas y nivel medio de educación en la provincia de Neuquén. El problema de la desigualdad educativa y la obligación legal de la universalización. En Mas Rocha, S.; Gorostiaga, J.; Tello, C. y Pini, M. (comps.) La educación secundaria como derecho. Buenos Aires: La crujía Ediciones. 
- Fernández, N. (2013). Los vínculos entre educación y trabajo: acerca de los saberes en la formación para el trabajo en estudiantes y egresados de escuelas medias de la Provincia de Neuquén. En Hernández, A. y Martinez, S. (coord.) Investigaciones en la escuela secundaria: Política y Trabajo. Río Negro: Publifadecs.

- Grinberg, S. (2014). La educación en tiempos de gerenciamiento. La escuela entre el 'hazte a ti mismo' y la lucha por estar. En Pini, M.; Mas Rocha, S.; Gorostiaga, J.; Tello, C. y Asprella, G. (coord.) La escuela secundaria ¿modelo en reconstrucción? Buenos Aires: Aique Ediciones.

- Hernández, A.; Machado, L.; Moschini, G.; Roldán, S. (2014). Desafíos de la obligatoriedad en la escuela secundaria: una mirada de proyectos escolares en la Provincia de Neuquén. En I/ Reunión Científica: La investigación sobre educación secundaria en Argentina. Buenos Aires.

- Moschini, G. (2013). Nuevas configuraciones en el Nivel Secundario. Perspectivas teórico metodológicas para el análisis de alternativas escolares destinadas a la inclusión de jóvenes de la provincia de Neuquén. VI Congreso Nacional y IV Internacional de Investigación Educativa. Facultad de Ciencias de la EducaciónUniversidad Nacional del Comahue.

- Rockwell, E. (1995). De huellas, bardas y veredas: una historia cotidiana en la escuela. En Rockwell, E (comp.) La escuela cotidiana. México: Fondo de Cultura Económica.

\section{Normativas}

- Ley de Educación Nacional 26.206/06.

- Ley de Educación Técnico Profesional 26.058/05.

- Resolución 088/09 Consejo Federal de Educación.

- Resolución175/12 Consejo Federal de Educación.

- Resolución 188/12 Consejo Federal de Educación.

- Resolución 1687/11 Consejo Provincial de Educación de Neuquén.

Resolución 0362/10 Consejo Provincial de Educación de Neuquén. 